\title{
Presentation the new surgicaly approach in the treatment of pediatric lung hydatid disease non- captonage procedure without closure of the communication bronchial opening
}

\begin{abstract}
Introduction: Treatment of pediatric lung hydatid disease is primarily surgical, and any suspicion on hydatid lung disease justified indication for surgical intervention. The aim of our research is to investigate efficacy and safety of treatment of residual cavity by "non-capitonage" method, without closure of communicating bronchial opening compared to the classic "capitonage" method with closure of communicating bronchial opening.
\end{abstract}

Patients and Methods: The study included 80 patients of both sexes under aged 18 years, who were surgically treated at the Clinic for Child Surgery and the Department of Thoracic Surgery, Clinical Center University in Sarajevo (KCUS) and Childrens Surgical Clinic of the Institute of Children's Diseases, Clinical Center of Montenegro in Podgorica due to pulmonary echinococcosis, based on clearly defined criteria for inclusion and exclusion from the study. 40 patients (group A) were subjected to noncapitonnage treatment of residual cavity that remained after the removal of parasites and partial pericystectomy without closure of orificium of bronchial opening. The control group consisted of 40 patients who had done narrowing of residual pericystic cavity with closure of bronchial opening.

This is a multicenter, open-targeted clinical comparative study of safety of surgery procedures and intraoperative findings. Important aspects of these procedures being assessed included: the duration of the surgical procedure, the amount of administered blood products, drainage length, the amount of secretion, duration of drainage fistula, the time required for reexpansion of lungs, and variables of complications (such as : occurrence of empyema, atelectasis, postoperative fever, postoperative wound infection). Postoperative effectiveness (efficacy) was measured by a record of length of stay in the intensive care unit and hospital stay, as well as radiological findings of the subjects lungs six months after surgery.

Results: In the control group we observed that the overall time of surgery was longer compared to the study group. Man-Vitni's U test confirmed a statistically significant difference between the results of the test and control groups, $U=285.50, z=4.957$, $\mathrm{p}=0.0001$. Additionally, we observed a slightly larger amount of fluid drained in the control group vs. the study group. Man-Vitni's U test revealed a statistically significant difference in the amount of drained fluid between the study and control groups, $\mathrm{U}=325.50, \mathrm{z}=4.583, \mathrm{p}=0.0001$. In the study group there were no cases of postoperative pulmonary atelectasis, while in the control group were $16(40.0 \%)$ patients with documented atelectasis. Mean time to reexpansion of the lung using the control radiological findings was 11 days in the study group $(\mathrm{Md}=11.000$ days, $\mathrm{n}$ $=40)$ vs. 16 days in the control group of patients $(M D=16.000$ days, $n=40)$. We also observed that the control group of subjects had significantly longer time in the intensive care unit compared with the experimental group $(\mathrm{U}=426.00, \mathrm{z}=3.654$, $\mathrm{p}=0.0003)$. Subjects in the control group had significantly longer hospitalizations compared with the experimental group $(\mathrm{U}=373.50, \mathrm{z}=4.112, \mathrm{p}=0.0001)$ as well.

Conclusions: We report here meaningful differences in two surgical approaches to the managamanet of hydatid disease in our medical system. Non-capitonnage surgical methods resulted in a significantly shorter duration of surgery, smaller drainage of secretions, atelectasis and pleural empyema events, shorter time required for reexpansion of the surgically intervened lungs and better overall outcomes, vs. the control surgical approach 6 months postoperatively. In our experience, noncapitonnage surgical methods compared much more favorably when compared to the capitonnage method, as reflected in the significantly shorter postoperative stay of patients in the intensive care unit and in hospital. The treatment of any residual pericystic cavity of pulmonary echinoccocus in children, especially if it is a giant, complicated and multiple hydatid cysts should be the method of "non-closure" or "nonarrowing" (non-capitonnage) due to the rapid overlaying of residual pericystic cavity by epithelial cells originating from surrounding pleura and lung parenchyma
Volume 6 Issue I - 2019

Karavdić K,' Mehić B, ${ }^{2}$ Guska S, ${ }^{3}$ Bajmak $^{4}$

'Clinic for Pediatric Surgery, Clinic Center of University Sarajevo, Bosnia and Herzegovina

${ }^{2}$ Clinic for Lungs Diseases, Clinic Center of University Sarajevo, Bosnia and Herzegovina

${ }^{3}$ Clinic for Thoracic Surgery, Clinic Center of University Sarajevo, Bosnia and Herzegovina

${ }^{4}$ Children's Surgery Clinic, Institute for Diseases of Children, The Clinical Center of Montenegro, Podgorica and Montenegro

Correspondence: Karavdić K, Clinic for Pediatric Surgery, Clinic Center of University Sarajevo Bosnia and Herzegovina, Bosnia and Herzegovinaa, Email kenan.kv@bih.net.ba

Received: September 18, 2018| Published: February 28, 2019

Keywords: capitonage, hydatid disease, bronchial opening 


\section{Introduction}

Echinococcosis (hydatidosis) is a widespread, parasitic zoonosis caused by echinococcus trousers (Echinococcus granulosus or Echinococcus multilocularis) where humans are a transient host and develop a larval form of the parasite. Echinococcosis is an endemic disease for the Mediterranean region, South America, Australia, New Zealand, Middle East, Alaska and Canada. ${ }^{1,2}$ The annual incidence of the disease varies from less than 1-220 per 100,000 inhabitants to different endemic areas. Incidence per 100,000 inhabitants (2002 data) varies from Uruguay 6.2 Spain 6.2 Turkey 4.4 Chile 4.0 Italy 1.9 Argentina 1.42 Peru 1.1 Australia 0.23. ${ }^{2-4}$

In 1996, the World Health Organization (WHO) published a a guidance for the treatment of pulmonary echinococcosis. The International Working Group on Education (IWGE), the World Health Organization (WHO), in 2010, adopted a consensus on echinococcosis treatment supported by a panel of experts. ${ }^{5-7}$ The treatment is primarily surgical, and any suspicion of hydatine shading of the lungs documented by chest x-ray, justifies an indication for surgical intervention. Urgent surgical treatment is needed in patients with predictive cyst rupture, compromised by vital organs with mass effect, haemoptysis, secondary infected cysts and secondary, infection due to obstruction and pain. ${ }^{8}$

The surgical procedure for treating hydatidic disease may be conservative or radical. In pediatric thoracic surgery, conservative surgical procedures are mainly used because of the recuperative ability of pulmonary parenchyma in childhood. ${ }^{9}$ The goal of the current, so called "conservative" surgical treatment of lung hydatidal disease is the eradication of parasites, the removal of the endocyst without intraoperative rashes, and the closure of the pericystic cavity with the maximum preservation of the pulmonary parenchyma. There is no direct fusion between the cyst and pulmonary parenchyma, so that all uncomplicated cysts can always be separated from the lungs. After removing the hydatid cyst, the bronchial fistula can be closed and the pericystic cavity is obliterated by the prevailing secondary infection. The pericystic wall, in fact, is composed of compressed athectic pulmonary tissue produced by the growth of the endocyst. A bronchial opening occurs when a bronchus moves through the growth of hydatid cyst and thus produces a fistulous communication between the bronchial and the cystic cavity.

\section{Patients and methods}

The study included 80 patients of both sexes aged under 18 years, who were surgically treated at the Clinic for Pediatric Surgery and the Clinic for Thoracic Surgery, Clinical Center University in Sarajevo (KCUS) and Childrens Surgical Clinic of the Institute of Children's Diseases, Clinical Center of Montenegro in Podgorica, due to pulmonary echinococcosis. All surgical cases included in this case series were diagnosed based on clearly defined criteria for inclusion and exclusion from the study. Forty patients (group A) were subjected to non-capitonnage treatment of the residual cavity that remained after the removal of parasites and partial pericystectomy without closure of orificium of the bronchial opening. The control group consisted of 40 patients who underwent surgical narrowing of the residual pericystic cavity with closure of the bronchial opening.

This is a multicenter, open-targeted clinical comparative study of safety of surgery procedures and intraoperative findings: the duration of the surgical procedure, the amount of administered blood products, drainage length, the amount of secretion, duration of drainage fistula, the time required for reexpansion of the operative lungs, and variables of complications such as: occurrence of empyema, atelectasis, postoperative fever, postoperative wound infection were all collected and analyzed.. Postoperative effectiveness (efficacy) was followed by a record of length of stay in the intensive care unit and hospital stay, as well as finding the control radiological findings in these surgical lungs six months after surgery.

Adjunctive chemotherapy before and after surgery is applied in all patients to reduce the risk of relapse by inactivating the protoscolysis and to reduce the tension of the cyst due to its easier removal. The therapy typically starts 4 days before surgery and continue 1-3 months later. Albendazole is given at a dose of $10-15 \mathrm{mg} / \mathrm{kg}$ body weight per day, in two separate doses, and the usual dose is $800 \mathrm{mg}$ per day. Therapy is most often indicated for a minimum of 3-6 months.

\section{Surgical treatment}

The patient is placed in the lateral decubitus position. The procedure is performed under general anesthesia with a separated endotracheal intubation with ipsilateral lung collapse. The classic posterolateral thoracotomy is performed through the V or VI intercostal space (Figure 1). The main objectives of the surgical treatment are eradication of parasites, prevention of intrathoracic rupture, eradication of the cystic components, preserving the lung parenchymal as much as possible, avoiding the reaction and preventing contamination during surgery. For this purpose, the surrounding tissue around the cyst is protected with a gas soaked in $10 \%$ Povidine-iodine solution or hypertonic $\mathrm{NaCl}$ to prevent possible dissemination (Figure 2).

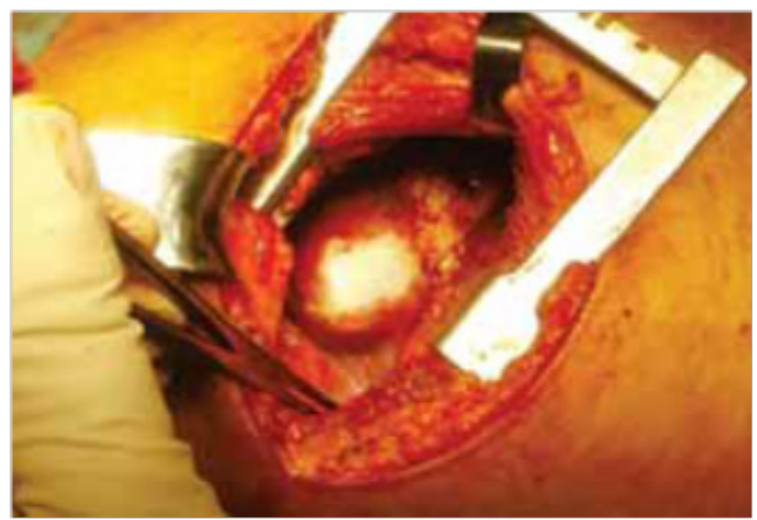

Figure I Posterolateral Thoracotomy through VI i.s.

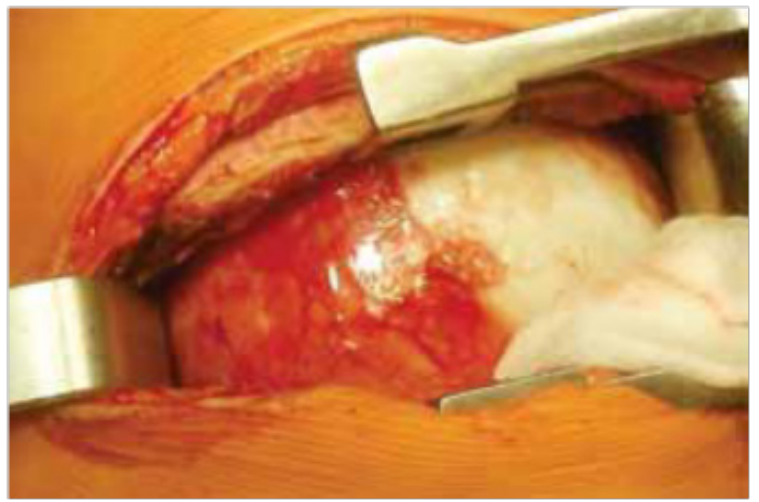

Figure 2 Protecting surrounding tissue by a gas soaked with hypertonic $\mathrm{NaCl}$.

Using the aspiration needle, the hydatid cyst is aspirated through the most distorted part, (Figure 3) and the same amount of $10 \%$ 
povidone-iodine is injected into the cyst. Waiting for 10 minutes, and then the most prominent part of the cyst is opened with scissors or electrocautery, (Figure 4) and the endocyst is removed with forceps or another instrument (Figure 5). After that, the residual cavity is irrigated with a $10 \%$ Povidine-iodine solution which is subsequently aspirated by a suction apparatus. A partial resection of the wall of a pericyst with scissors is made (Figures $6 \& 7$ ). Examination group is treated with a non-capitonnage method and not by closing the orifice of the corresponding bronchus in the periclinic cavity Image (Figures $8-10)$.

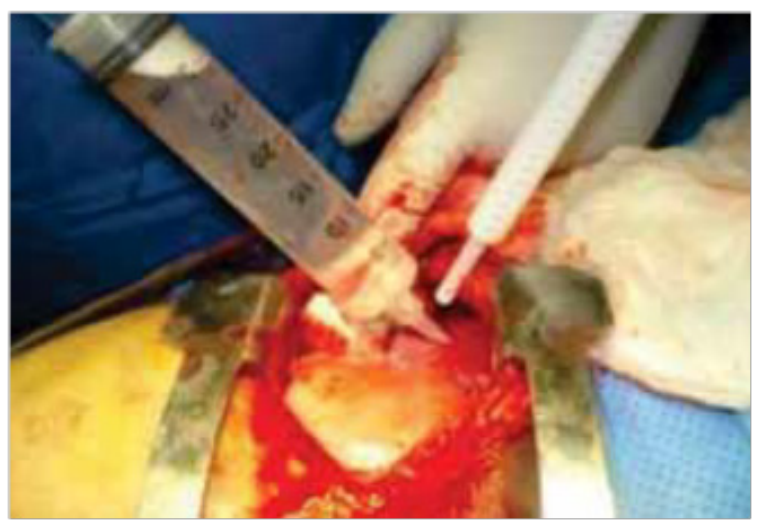

Figure 3 Aspiration of the hydatid cyst through

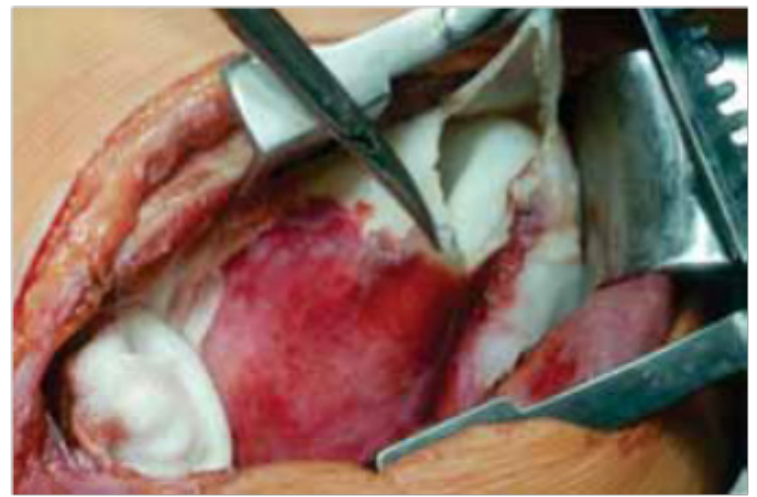

Figure 4 The most prominent part of the cyst is opened the most distorted part of the scissors

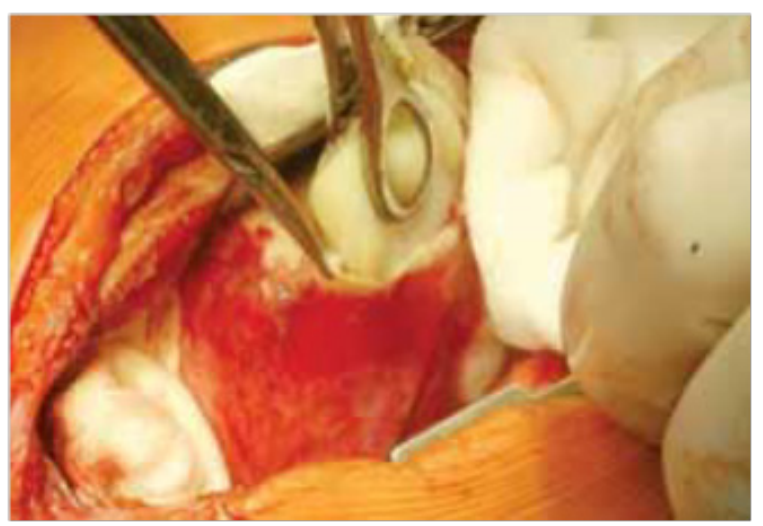

Figure 5 endocyst

The control group is treated with the capitonnage method of the pericidal cavity and the closure of the orifice (Figure 11). The Orificium of the communicating bronch closes after its verification with 3-0 Polyglactin suture. After closing the bronchial opening, it is carefully determined whether there is an air bubble by applying a physiological fluid to the cavity during the re-expansion of the lungs. The residual pericystic cavity is obliterated with individual sutures, starting from the deepest level to the surface using 3-0 polyglactin suture (Vycril).

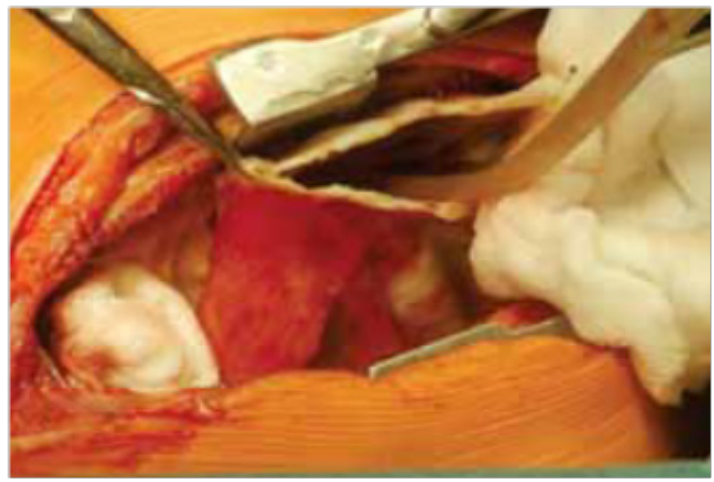

Figure 6 Partial resection of the wall of a pericyst with scissors-I.

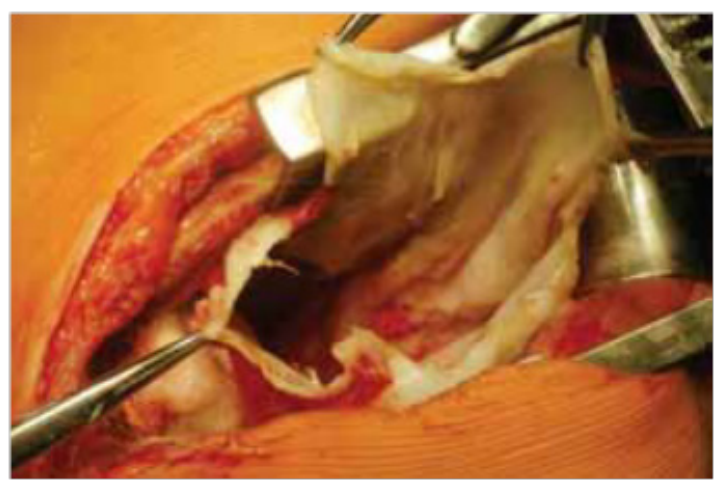

Figure 7 Partial resection of the wall of a pericyst with scissors-2.

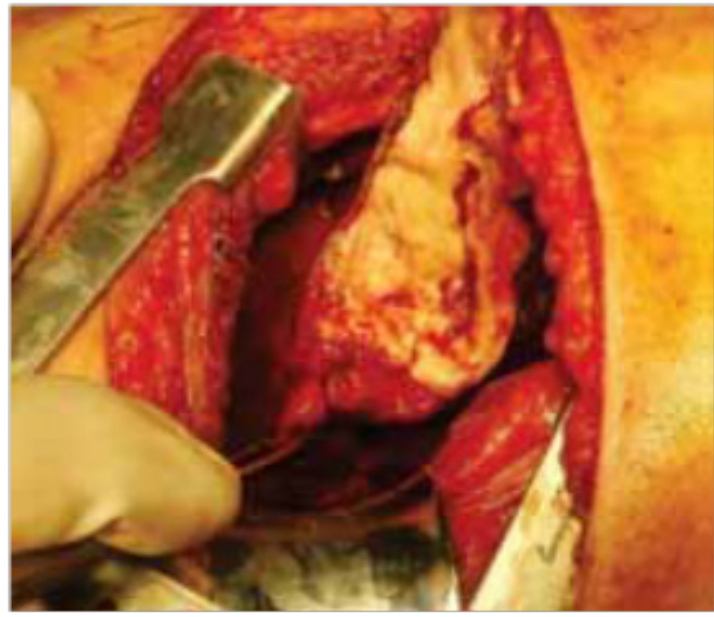

Figure 8 Closure of the pericystic cavity.

During postoperative treatment, which is identical for both examinees and the control group, thoracic drains are at -20 to $-25 \mathrm{cmH} 20$ pressure and are removed when no air fistula is recorded, when fluid drainage is less than $150 \mathrm{ml} / 24 \mathrm{~h}$ and when a radiological improvement was confirmed.

After discharge, all patients were treated with X-ray after 6 months of primary therapy. The criteria for radiological improvement were 
the loss of shaded cysts, and the resorption of postoperative pericystic inflammation and pleural reaction (Figure 12-15).

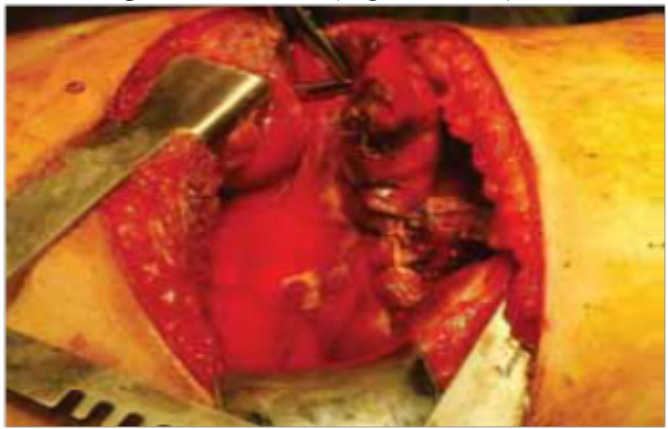

Figure 9 Pericystic cavity covered non-capitonnage method (partial surrounding lung tissue pericystectomy, sutura pericitis and no closing the communicating bronchial opening).

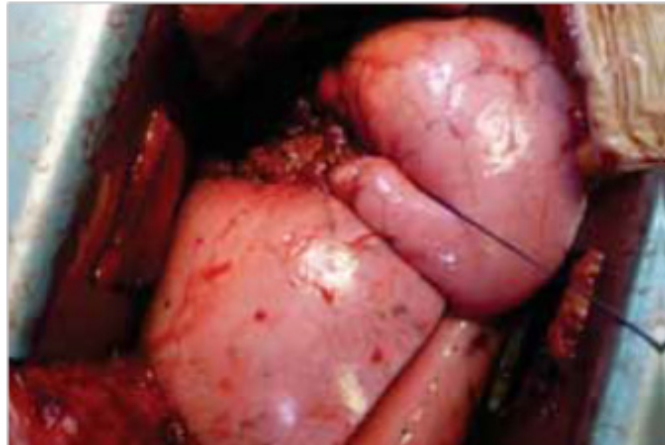

Figure 10 Reexpanded lungs at the end of the surgery.

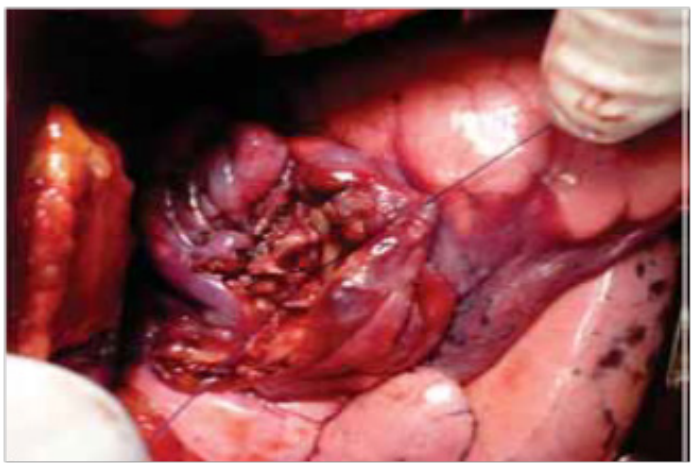

Figure II Capitonnage method.

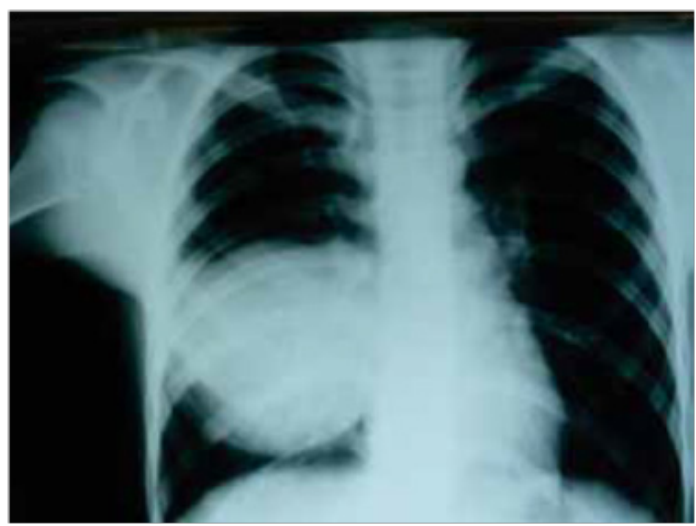

Figure $\mathbf{2}$ Preoperative $\mathrm{x}$-ray, right round shadow.

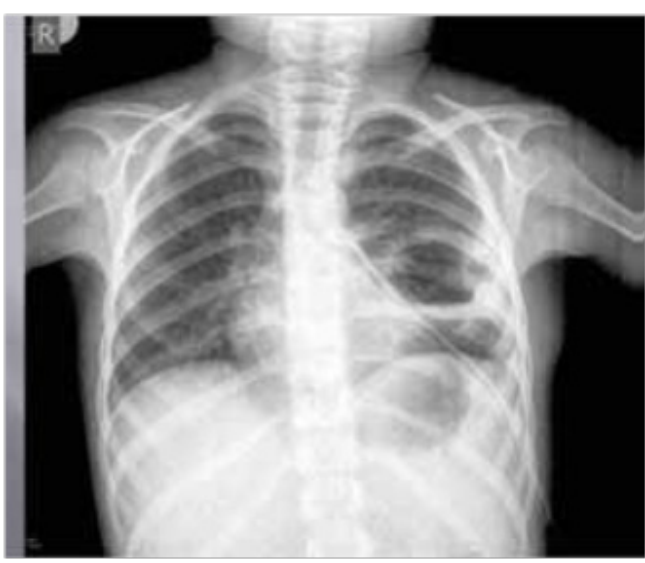

Figure I 3 Postoperative x-ray, pericystic cavity non-capitonnage method.

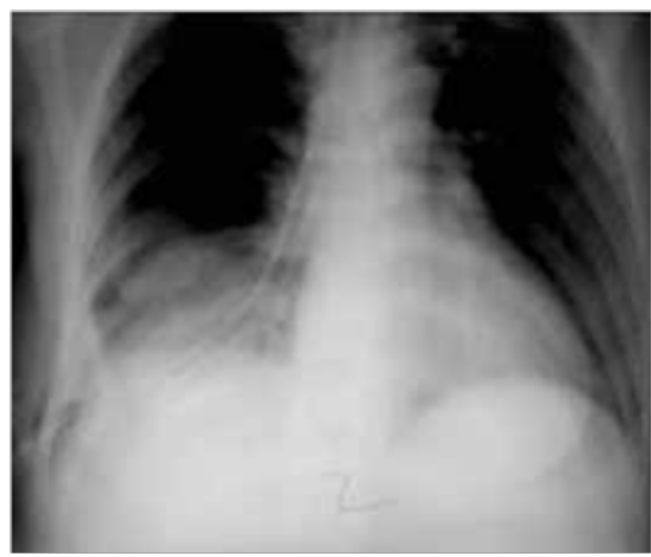

Figure I4 Postoperative x-ray cavity capitonnage method.

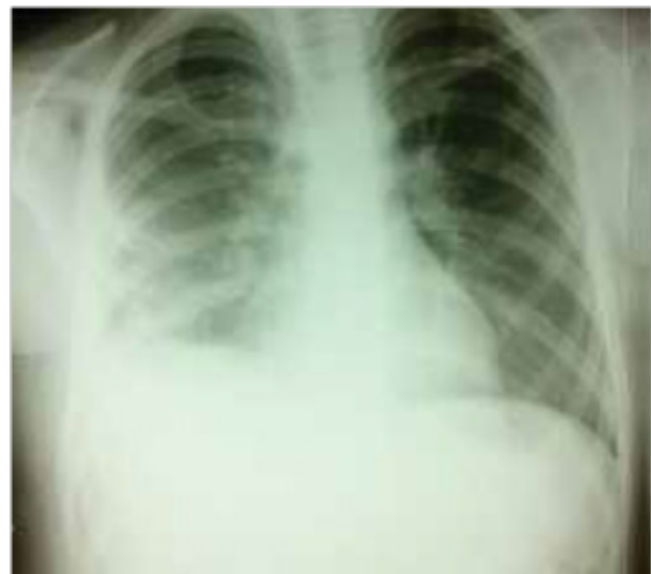

Figure 15 X-ray finding after 6 months loss of shaded cyst and resorption of pericystic inflammation and pleural reactions.

\section{Results}

The average age in the examined group was $10.2 \pm 4.5$ years, and in the control period of $8.3 \pm 2,7$ years. The youngest patient in the examined group was 4 years old and the oldest was 18 years old, while in the control group, the youngest was 3 years old, and the oldest 16 years old. Hi square test showed a statistically significant difference in distribution based on the use of blood derivatives during surgery. There were more patients in the control group who received blood 
products than in the examined group; $\chi 2(1, \mathrm{n}=80)=5.003, \mathrm{p}=$ $0.0253, \mathrm{~K}=0.243$ (Chart 1). The results shown in Chart 2 show the mean duration of the surgery in the examined group $(\mathrm{Md}=120,00$ minutes, $\mathrm{n}=40)$ and the control group $(\mathrm{Md}=190,00$ minutes, $\mathrm{n}=$ 40). In the control group, surgical procedures lasted longer than the test group. Man-Vitniy The test confirmed a statistically significant difference between the results of the examined and the control group, $\mathrm{U}=285.50, \mathrm{z}=4.957, \mathrm{p}=0.000$. Man-Vitniy The test confirmed a statistically significant difference between the results of the examined and control group, $U=285.50, z=4.957, p=0.0001$. The results in Chart 3. show a mean time to the reeexpansion of the lung based on the lung control test (CXR confirmation) for the examined $(\mathrm{Md}=$ $11,000$ days, $\mathrm{n}=40)$ and the control $(\mathrm{Md}=16,000$ days, $\mathrm{n}=40)$ group of subjects. A slightly higher number of days have been reported to achieve lung resection in the control group (Chart 4). Man-Vitniy In the test, a significant difference in the number of days to achieving lung re-expansion between the examined and the control group was found, $U=397.00, z=3.885, p=0.0001$. Man-Vitniy The test revealed a statistically significant difference between the length of stay in the intensive care unit of the examined and control group, $U=426,00, z$ $=3,654, p=0,0003$. The same test revealed a statistically significant difference in the length of hospital treatment, between the examined $(\mathrm{Md}=11,500$ days, $\mathrm{n}=40)$ and the control group $(\mathrm{Md}=16,000$ days, $\mathrm{n}=40), \mathrm{U}=373,50, \mathrm{z}=4,112, \mathrm{p}=0.0001$, (Chart 5).

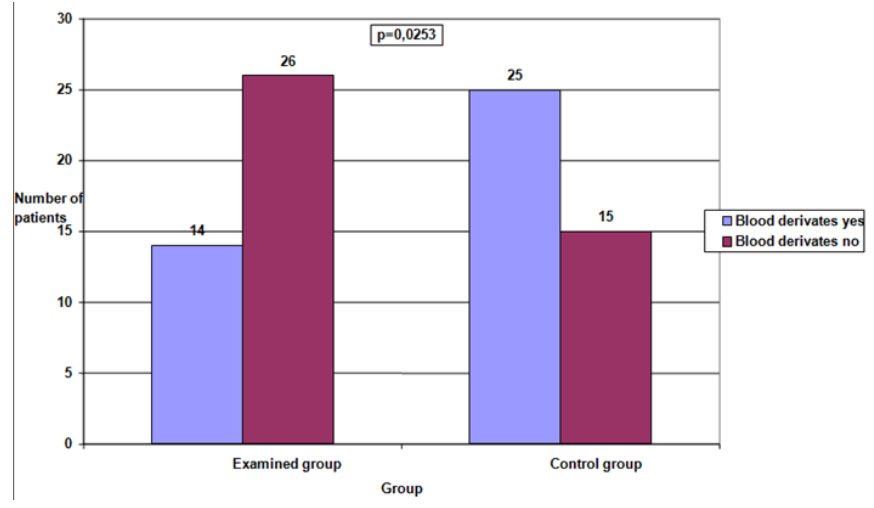

Chart I Distribution based on the use of blood products.

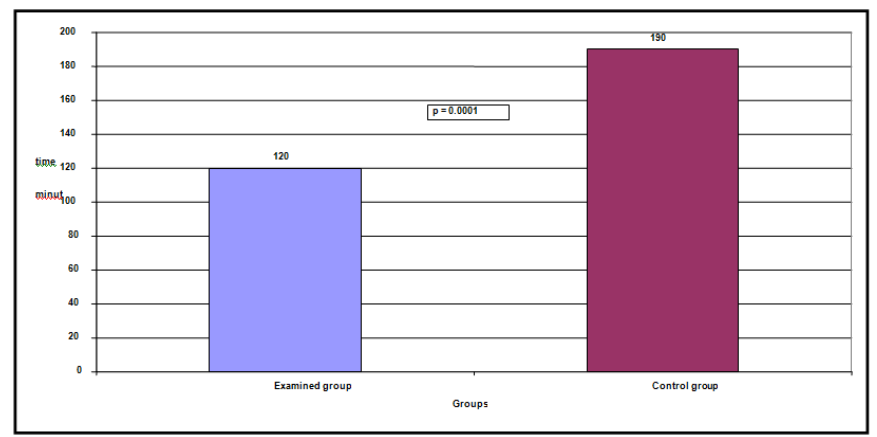

Chart 2 Mean duration of surgery for both groups of subjects.

Hi quadrat logrank test showed a significant difference between the total length of treatment for both groups. The analysis showed that the overall length of treatment was statistically significantly shorter in the examined group; $\chi^{2}(1, \mathrm{n}=80)=13.7406, \mathrm{p}=0.0002$. In the examined group, median time was 11 days, and in the control group 16 days. None of the groups recorded an adverse outcome.

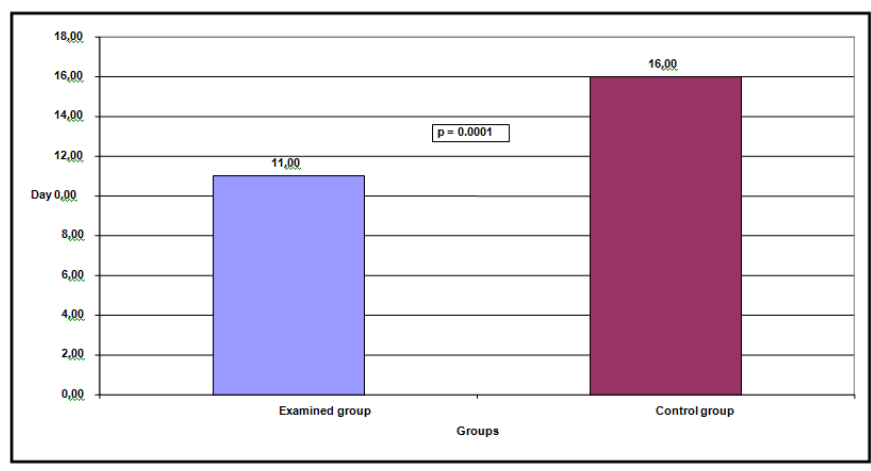

Chart 3 The average number of days to reexpansion of the lungs for both groups.

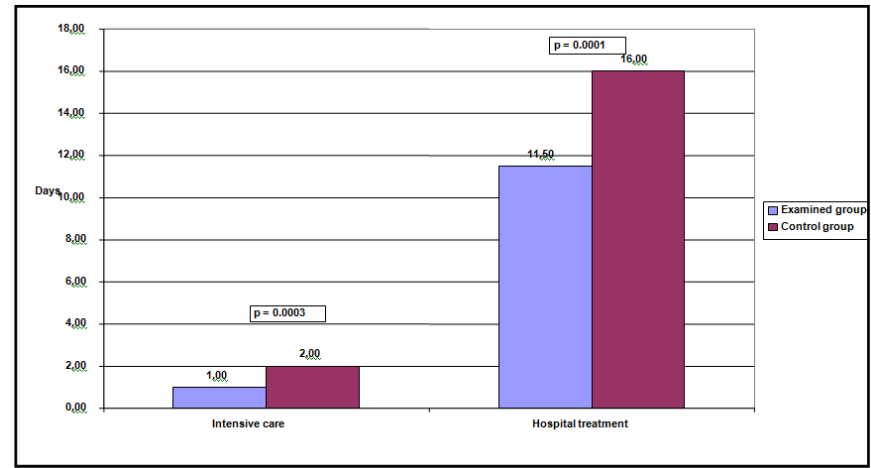

Chart 4 Mean length of stay in intensive care unit and duration of hospital treatment (days) for both groups of subjects.

\section{Discussion}

Echinococcosis is the most severe parasitic zoonosis and is still a national problem in endemic areas such as the Mediterranean parts of Bosnia and Herzegovina and Montenegro that require continuous research work in both diagnostic and therapeutic terms. The disease is particularly prevalent in underdeveloped countries and in countries where nomadic cattle breeding is represented, and the World Health Organization (WHO) has recognized the disease as extremely dangerous for such poorer areas. WHO issued the 1996 Guide to Procedural Guidelines, and in 2011 formed the IWEG (International Working Education Group) with the aim of providing the guidelines for proper procedures and adequate treatment for this disease. ${ }^{10}$ The latest surgical techniques insist on conservative surgical treatment and preservation of pulmonary parenchyma as much as possible. Radical surgical methods (segmentotomy, lobectomy, pneumonectomy) are avoided, although they also have indications. Ozer et al., ${ }^{11}$ Concludes that pulmonary resection should not be routinely applied to hydatidic lung cysts and to relatively young people living in endemic areas where they may be infected again. ${ }^{12}$

In countries where the hydatidic disease is endemic, as in Turkey, resection should be avoided as much as possible because the disease can recur in patients treated surgically for pulmonary echinococcosis. The goal of the current conservative surgical treatment of the hydatidic lung disease is the eradication of parasites, the removal of the endocyst without intraoperative rashes, the treatment of the pericystic cavity with the maximum preservation of the pulmonary parenchyma, and the closure of the communicating bronchus. A pericystic wall 
is compressed resulting in atelectectic pulmonary tissue caused by parasite growth (endocysts). The bronchial opening is in fact a small lateral fistulous communication between the bronchus and the pericystic cavity caused by the growth of the cyst and the bronchial movement. ${ }^{13}$

The main dilemma is how to treat the remaining pericystic cavity and bronchial communicate opening after removal of the endocyst. In the middle of the 20th century there were techniques of endocysts enucleation, some authors recommend the complete removal of pericystic atelectectic tissue, which can lead to complications (bleeding, air fistulae). ${ }^{14}$ Although first introduced in 1899 by French surgeon Pierre Delbet, the treatment of residual pericystic cavity with capitonnage (closure-narrowing) became commonly accepted in the 1960 s with modification by Crausaz. A number of authors, especially from hyperendemic areas, consider that the pericistyc residual cavity can be left open and capitonnage performed only with the closure of the communicating bronchial opening. ${ }^{11}$

There is very little information regarding the duration of the above mentioned surgical procedures. In an available comparative study, it was concluded that the duration of capitonnage during surgery was at most 3-5 minutes, in case of smaller residual pericystic cavities, and that the duration may be longer in case when cysts were giant or complicated. ${ }^{15}$ Based on this case series reported here, we conclud that the method applied on the examined group is safer with respect to its duration. The biggest difference noted was due to the length of the procedure applied to the control group that requires the visualization of the communicating bronchus ( $\mathrm{NaCl}$ test), the careful closure of the communicating bronchus with 3-0 polyglactin suture and control test with $\mathrm{NaCl}$ which requires the re-expansion of the pulmonary parenchyma. An additional circumstance that influenced the statistically significantly longer duration of surgery in the control group (in which the capitonnage method was used) was in large part due to the finding that that $20 \%$ of these patients had giant cysts and $40 \%$ had complicated cysts. We believe that this dataset supports the use of the non-capitonage surgical procedure in patients with complicated and gigantic cysts.

Also, by examining data related to the safety of the operative procedure, the use of blood products during the surgery was considered. No similar research was found in the searched literature. Our results showed a statistically significant difference in distribution based on the use of blood derivatives during the surgical procedure. The reasons for an insignificant difference in the amount of blood products during surgery are most likely to be sought in small-vessel injuries that lie along the pericystic wall of the cavity during the capitonnage procedure and during the closure of the communicating bronchus. Eren support non-capitonnage technique because of the possibility of damage to blood vessels during the capitonnage method, especially in large and complicated cysts. ${ }^{16}$

There were no cases of thoracic empyema in the investigated group, while in the control group there were 13 (32.5\%) cases with empyema. There were no cases of lung atelectasis in the examined group, while $16(40.0 \%)$ of subjects with lung athelectasis were observed in the control group.

In the investigated group, ten patients $(25 \%)$ were observed following treatment with elevated body temperature, while in the control group there were $16(40.0 \%)$ subjects with elevated body temperature. There were no cases of wound infection in the investigated group, while in the control group there were $3(7.5 \%)$ respondents with wound infections, which proved to be statistically insignificant.

Post-operative follow-up included data on efficacy such as: record of length of hospitalization, length of stay in the intensive care unit, number of days required to record radiological improvement (reexpansion)

Caushi and colleagues compared two groups of patients with pulmonary hydatid disease, where they treated the residual peristatic cavity with capitonnage and the non-capitonnage method. They generally agree that the most important point in the treatment of residual pericystic cavity is the closure of the bronchial opening. Caushi and the authors in both groups examined all the bronchial openings after endocystectomy. They concluded that the capitonnage method did not provide an advantage in the length of hospitalization, the length of stay in intensive care, the duration of the airway and the length of the chest drainage or the prevention of complications such as empyema, recurrence or prolonged air fistula. They concluded that the residual cavity is covered with pleura, and that the capitonnage method is not necessary. ${ }^{17}$

Demirhan and his associates announced that the capitonnage method does not represent any advantage over the duration of hospitalization, stay in the intensive care unit, duration of air fistula and thoracic drainage or the prevention of complications (empyema, recurrence, or prolonged air fistula).

The results of this study showed a significantly shorter hospitalization and stay in the intensive care unit, less drained fluid, significantly less occurrence of atelectasis in the examined noncapitonnage group. There was no statistically significant benefit of non capitonnage in relation to capitonnages when it comes to the duration of chest drainage and wound infection. Usluer and associates found that capitonnage can cause an atelectasis by obliterating the bronchus surrounding the pericyst, Without capitonnage, presumably, the wall of the peristatic cavity is covered by the epithelial cell, and for an uncertain length of time. They concluded that experimental studies would be required to test this hypothetical possibility. ${ }^{18}$ Dakak have concluded that in cases of large peripheral cysts in children, capitonnage may limit lung expansion. ${ }^{19}$

With positive pressure during anesthesia, capitonnage seams can lead to laceration of the lung tissue, especially in complicated cysts. Capitonnage seams can damage the blood vessels and bronch, resulting in bleeding and atelectasis. Capitonnage has been published as a procedure that has no preference for long-term and can lead to distortion of the residual lobe or lungs. ${ }^{20}$

In further research we found publications where the authors performed the capitonnage method only in small cysts. Although higher operative comorbidity was found in non-capitonnage patients, re-operation due to complications was only needed in capitonnage cases. In these cases, operators had more difficulty in closing bronchial openings in infectious and perforated cysts. However, these complications did not contribute to longer hospitalization or duration of thoracic drainage. ${ }^{21}$ Other complications have been withdrawn by a general treatment with little effect on the length of hospitalization. Following non-capitonnage patients postoperatively, they noticed that the cystic cavity completely disappeared in the lung recording within a maximum of 2 weeks after surgery due to the lung's ability to expand. 
Based on these results, it was concluded that capitonnage is not a basic surgical treatment of pulmonary hydatid cysts. Careful closure of the bronchial opening, in their opinion, should reduce morbidity.

Our own surgical outcomes reported here match the results of the available research. The treatment of complicated, multiple and giant cysts has proven to be more efficient and safer than the noncapitonnage method. The reasons lie most likely in the fact that surgeries on large cysts increase the possibility of bleeding, air fistula or atelectasis precisely because of the large surface that must be narrowed and closed. Also, stitches placed by the capitonnage method on complicated (perforated and infectious) cleanses can lead to secretion or release of seams by which capitonnages are made or which are bubbled..$^{22,23}$ By searching through available literature, we found the results of comparative studies of pediatric patients with complicated and uncomplicated lung cysts where the authors concluded that the capitonnage procedure had no advantage and did not recommend it as unnecessary. ${ }^{24,25}$

In cases of giant cysts in children, capitonnage may limit lung resection. ${ }^{26}$ The authors concluded that capitonnage should only be performed on small cysts and only after the cystostomy only the bronchial opening was induced. Although the authors had higher morbidity in non-capitonnage patients, reoperation due to complications were performed only in capitonnage patients. Larger complicating the postoperative morbidity in patients non-capitonnage is attributed to relaxation or releasing the stitches positioned on bronchial opening in patients infected with perforated or cyst. ${ }^{27}$

Similar to the results of the analyzed papers, our results showed full lung re-expansion in the first two weeks. There was no significant difference in radiological improvement between the examination and control group. Particularly in patients with perforated cysts, the rate of postoperative complications may be reduced by a cystostomy procedure by closing the bronchial opening without the capitonnage method. ${ }^{28,29}$ Our study showed a significantly lower number of patients in the non-capitonnage compared to the control group who had atelectasis in the postoperative period as complications. Sokouti and authors consider that capitonnage does not provide any advantage in comparison with patients with whom capitonnage has not been performed. ${ }^{30}$

There is a large number of publications by the advocate of the capitonnage of residual percicystic cavity after the removal of the endocyst. Yaldiz reported that capitonnage shortens postoperative retention of thoracic tube and reduces morbidity compared to patients without capitonnage (pediatric population of 15 patients). ${ }^{31}$ Eser et al., ${ }^{32}$ Evaluated 236 patients with pulmonary echinococcosis treated so called parenchymal protective surgery by comparing patients with the capitonnage and non-capitonnage method. The parameters of safety (duration of drainage) and parameters of efficiency (length of hospitalization) were followed. They concluded that better results were seen in patients with capitonnages and considered the necessary part of the procedure.

Nabi and associates applied a capitonnage method to all patients with minimal complication rates. They insisted on the careful and minuscule capitonnage operative technique, believing that in this way they could avoid possible complications such as bleeding, air fistula proliferation and atelectasis. ${ }^{33}$ Helerezoglu and associates concluded that capitonnage is a better technique because it creates another barrier against prolonged air fistula in the prevention of bronchial fistula. In their study there was no significant difference in patients with capitonnage and non-capitonnage method, except in patients with giant and complicated cysts (prolonged air fistula, length of hospitalization) with significantly lower results. ${ }^{34}$

In the literature we did not find data on the closure of the communicating bronze as a procedure. What's more, in all published papers dealing with the topic of residual percussion cavity treatment, it is insisted on the closure of the communicating bronze. Eser and associates explain a slightly higher rate of prolonged fistula in their series because the bronchial opening remained unnoticed during the surgical procedure due to visual problems of blood clots and secretion as causative factors in this condition. Eser pointed out that the closure of the bronchial opening is very important. ${ }^{35}$

Knowing the pathology of bronchial communication (the growth of endocysts is bronchial, even when the large formation of small fistulous bronchial communication with periclinic cavity), we felt that such a small fistulous communication most likely has the ability to spontaneous closure, i.e. the surrounding epithelium and the parietal pleura filling the cavity of an uncontaminated percussion cavity covers and obliterates the fistulas opening. Especially in pediatric patients, the ability to regenerate the epithelium is high. We found the justification for this attitude especially in patients with large and complicated cysts where additional application of seams could lead both to the method of capitonnage to the injury of neighboring bronchi and the emergence of new fistula or atelectasis as well as to the injuries of blood vessels with consequent haemorrhage. The results of this study justify this attitude (there is no significant difference in the duration of the air fistula and the occurrence of radiological improvement in patients who are closed by the communicating bronchus and in the patients in whom it is left open).

Of the 80 patients treated in this study, we did not have a single case of relapse. Considering the large number of complicated, gigantic and multiple cleaners in both groups, we consider this result satisfactory. The literature published a recurrence rate of $2-12 \%$ while Sayir and associates had a recurrence rate of $1.4 \% .{ }^{36}$ We further analyzed the finding of a control chest X-ray of the lung image 6 months after surgery. The success of the procedure was characterized by radiological improvement after 6 months. Radiological improvement was characterized by absence of atelectasis, loss of shadow operated cysts and resorption of postoperative cystic inflammation.

Based on the analyzed data of radiological recording in the investigated group, no subjects with lung atelectasis were recorded after surgery, while in the control group this complication occurred in 7 subjects. In the examined group, there was a disappearance of shadow of operable cyst in 31 subjects, while in the control group there were 20 respondents with shadow disappearing after 6 months. In the investigated group, there was a resorption of postoperative inflammation in 37 subjects, while in the control group there were 27 subjects who recorded resorption of postoperative pericystic inflammation. Following three radiological parameters, 6 months after surgery: the presence of atelectasis, the disappearance of shadow-operated cysts, and the resorption of postoperative periclinic inflammation, we concluded that the regression of radiological changes was significantly more pronounced in the test (capitonnage) compared to the control group (non-capitonnage).

Comparing the parameters related to the safety and efficacy of treatment of residual percussion cavity by non-closing or 
non-capitonnage without the need for the management of the communicating bronchus with parameters related to the safety and efficiency of the classical surgical method, we have received a clear confirmation of the advantage of this method. The safety of the method is reflected in a significantly shorter duration of surgery, significantly less use of blood derivatives and significantly less complications (atelectasis, empyema). Higher efficacy is reflected in post-operative follow-up, and this is markedly shorter hospitalization and stay in intensive care. Safety data on the duration of the extended air fistula, length of drainage, febrile and wound infection are not significantly different.

The choice of a definitive treatment in the surgical treatment of residual pericystic cavity and communicating bronchus is a topic of continuous discussion in world literature. This study follows the context and argumentation of these discussions by giving a new scientific contribution to the illuminating relationship of the noncapitonnage method without the discharge of the communicating bronchus and the procedure of the standard capitonnage method by treating the communicating lung echinosis of the lungs in children.

\section{Conclusions}

1. The safety of the non-capitonnage method is reflected in the statistically significantly shorter duration of the operation, statistically significantly lower drainage secretion, the number of empires and atelectasis, statistically significantly shorter time needed for lung resection, and in a statistically significantly better control after 6 months of surgery

2. The effectiveness (non-capitonnage) of the non-capitonnage method compared to the capitonnage method is reflected in the statistically significantly shorter postoperative stay of patients in the intensive care unit and statistically significantly shorter stay, retention of patients in the hospital.

3. Treatment of the pericystic cavity of echinocytes in children, especially in the case of gigantic, complicated and multiple hydatide cysts, should be a non-capitonnage method due to the rapid overlap of percutaneous cavity with epithelial cells surrounding the surrounding pleura and pulmonary parenchyma.

4. Treatment of residual percutaneous cavity with a non-capitonnage method without closing the communicating bronchus is an effective and safe method in the treatment of pulmonary echinocoses of children compared with the capitonnage method.

\section{Acknowledgments}

None.

\section{Conflicts of interest}

The author declares there is no conflicts of interest.

\section{References}

1. D'Alessandro A, Rausch RL. New aspects of neotropical polycystic (Echinococcus vogeli) and unicystic (Echinococcus oligarthrus) echinococcosis. Clin Microbiol Rev. 2008;21(2):380-401.

2. Howorth MB. Echinococcosis of Bone. Journal of Bone and Joint Surgery. 1945;27:401-411.

3. Stich A, Frosch M. Emergence of Polycystic Neotropical Echinococcosis. Emerging Infectious Disease. 2008;14(1):292-297.
4. Thompson RC. The taxonomy, phylogeny and transmission of Echinococcus. Exp Parasitol. 2008;119(4):439-446.

5. Fatimi S. Hydatid cyst disease of the thorax Reviews In Infection. 2010;1(1):42-48.

6. Simsek S, Balkaya I, Koroglu E. Epidemiological survey and molecular characterization of Echinococcus granulosus in an endemic area of eastern Turkey. Parasitol. 2010;172(3-4):347-349.

7. Dopchiz MC, Elissondo MC, Andresiuk MV, et al. Pediatric hydatidosis in the south-east of the Buenos Aires province, Argentina. Rev Argent Microbil. 2009; 41:105-111.

8. Ahmed ME, Abdelrahim MI, Ahmed FM. Hydatid disease, a morbid drop needs awareness. Sudan Med J. 2011;47(1).

9. Siracusano A, Teggi A, Ortona E. Human cystic echinococcosis: old problems and new perspectives. Interdiscip Perspect Infect Dis. 2009.

10. Brunetti E, Kern P, Vuitton DA. Writing Panel for the WHO-IWGE Expert consensus for the diagnosis and treatment of cystic and alveolar echinococcosis in humans. Acta Tropica. 2010;114:1-16.

11. Özer Z, Çetin M, Kahraman C. Pleural involvement by hydatid cysts of the lung. The Journal of Thoracic and Cardiovascular Surgery. 2009;123(3):495.

12. Ghosal J. Hydatid Lung Disease: An Analysis of Five years Cumulative Data from Kolkata. JLPI. 2012.

13. Moro P, Schantz PM. Echinococcosis: a review. Int J Infect Dis. 2009; 13:125-133.

14. Ekim H, Ozbay B, Kurnaz M, et al. Management of complicated giant thoracic hydatid disease. Med Sci Monit. 2009;15(12):15.

15. Fadhil GY, Amran AL. A surgical experience of 825 patients with thoracic hydatidosis in Iraq. IJTCVS .2008;24:124-28.

16. Gajbhiye AS, Sonak M, Meshram MM, et al. Surgical Management of Pulmonary Hydatid Cyst. Journal of Dental and Medical Sciences. 2013;6(12):59-64

17. Caushi F. Capitonnage or not? Which is the best operative technique for hydatid cysts of the lung; when should it be performed? Pneumon. 2011,24(2):177-181.

18. Usluer O. Surgical Management of Pulmonary Hydatid Cysts-Is Size an Important Prognostic Indicator? Tex Heart Inst J. 2010;37(4):429-434.

19. Dakak M, Caylak H, Kavakli K, et al. M Parenchyma-saving surgical treatment of giant pulmonary hydatid cysts. Thorac Cardiovasc Surg. 2009;57(3):165-168.

20. Rachid B, Amine B, Aziz C, et al. Giant viable hydatid cyst of the lung revealed by hiccups. The Pan African Medical Journal. 2012;13:48.

21. Cobanoglu U, Sayır F, Şehitoğlu A, et al. Therapeutic strategies for complications secondary to hydatid cyst rupture. Int J Clin Exp Med. 2011;4(3):220-226.

22. Akhtar J, Khan NA, Baneen U, et al. Giant pulmonary hydatid cyst mimicking elevated diaphragm: A diagnostic dilemma. Ann Afr Med. 2012;11:122-123.

23. Findikcioglu A. Necessity of Lung Resection in Neglected Cases of Pulmonary Hydatidosis. Ann Thorac Cardiovasc Surg 2010;16:187189

24. Dakak M, Caylak H, Kavakli K, et al. Parenchyma-saving surgical treatment of giant pulmonary hydatid cysts. Thorac Cardiovasc Surg. 2009;57:165-168.

25. Arroud M, Afifi MA, El Ghazi K, et al. Lung hydatic cysts in children: Comparison study between giant and non-giant cysts. Pediatr Surg Int. 2009;25:37-40. 
26. Sokouti M, Pezeshkian M, Ghabili K, et al. Surgical Procedures and Postoperative Complications in Patients with Giant and Non-giant Pulmonary Hydatid Cysts, Life Sci J. 2013 ;10(1):138-142.

27. Ghalab N. Giant viable hydatid cyst of the lung: a case report. Journal of Medical Case Reports. 2008;2:359.

28. Emirhan R, Onan B, Kiral H, et al. Surgical treatment of giant hydatid cysts in childhood. Turkish Journal of Thoracic and Cardiovascular Surgery. 2010;18:121-125.

29. Shrestha K, Shah S, Thulung S.Our Results in Surgical Treatment of Hydatid Cyst of the Lungs. PMJNPostgraduate Medical Journal of NAMS. 2010.

30. Yaldiz S, Gursoy S, Ucvet A, et al. Capitonnage Results in Low Postoperative Morbidity in the Surgical Treatment of Pulmonary Echinococcosis. Ann Thorac Surg. 2012;93:962-967.
31. Eser I, Gunay S, Cevik M, et al. Evaluation of 236 Patients with Pulmonary Cyst Hydatid Treated with Parenchimal Protective Surgical Treatment. Acta Medica Mediterranea. 2013;29:121.

32. Nabi MS. Pulmonary hydatid disease: What is the optimal surgical strategy? International Journal of Surgery. 20108(8):612-616.

33. Halezeroglu S, Okur E, Tanyü MO. Surgical management for hydatid disease. Thorac Surg Clin. 2012;22(3):375-385.

34. Sayir F, Çobanoğlu U, Şehitoğullari A, et al. Our eight-year surgical experience in patients with pulmonary cyst hydatidInt. J Clin Exp Med. 2012;5(1):64-71. 